\title{
Modelling Diffuse Interstellar Environments
}

\author{
Evelyne M. Roueff ${ }^{1}$ and Franck Le Petit ${ }^{1,2}$ \\ ${ }^{1}$ LUTH and UMR 8102 du CNRS, Observatoire de Paris, \\ Place J. Janssen, F-92190 Meudon, France \\ email: evelyne.roueff@obspm.fr \\ ${ }^{2}$ Onsala Space Observatory, S-439 92 Onsala, Sweden \\ email: franck.lepetit@obspm.fr
}

\begin{abstract}
Diffuse clouds are defined as low-density clouds permeated by the ultraviolet interstellar radiation field. We discuss the ensemble of atomic, ionic and molecular observations in the context of our Photon Dominated Region model. The comparison with all observational constraints is limited and implies that the diffuse environment is more complex than originally thought.
\end{abstract}

Keywords. ISM: abundances — ISM: molecules — molecular processes

\section{Introduction}

A number of observational challenges for chemical models of diffuse clouds is addressed in the advertisement of the present symposium. These include the explanation of high column densities of molecular ions such as $\mathrm{H}_{3}^{+}, \mathrm{CH}^{+}, \mathrm{HCO}^{+}$and polyatomic molecules $\mathrm{C}_{3}, \mathrm{CCH}$ and $\mathrm{c}-\mathrm{C}_{3} \mathrm{H}_{2}$. In addition, one may also emphasize the discovery of molecular nitrogen by Knauth et al. (2004), $\mathrm{HOC}^{+}$by Liszt, Lucas \& Black (2004) and the excitation of $\mathrm{H}_{2}$, which has received recent attention with the observations of the Far Ultraviolet Spectrograph Explorer (FUSE). Diffuse clouds are subject to ultraviolet radiation coming from the surrounding stars, which drives the atomic to molecular ratio via photophysical and photochemical processes. A. Sternberg in this volume has summarized the various physical and chemical aspects involved in Photon Dominated Regions (PDRs), which encompass diffuse clouds environments. We recall in $\S 2$ the main properties of our numerical code and define the physical parameters for a "standard" homogeneous diffuse cloud model. We discuss in $\S 3$ some aspects of diffuse clouds and compare model results with recent observations in $\S 4$. We present our conclusions and perspectives in $\S 5$.

\section{The "Meudon" PDR Code}

Our studies of diffuse clouds will be discussed in the frame of the "Meudon" PDR code which has been developed over more than 10 years (Abgrall et al. 1992; Le Bourlot et al. 1993; Le Petit, Roueff \& Herbst (2004); Le Petit et al. 2005) and which is downloadable on the website http://aristote.obspm.fr/MIS. The main distinctive features are:

- UV radiative transfer is solved by considering both dust absorption and discrete transitions of $\mathrm{H}_{2}, \mathrm{HD}, \mathrm{CO}$ and its isotopes. Explicit calculations of photodissociation probabilities are performed for these particular species. The impinging radiation field can be introduced for 1 or 2 sides of a plane-parallel slab of gas. The presence of a close bright star on one side of the cloud can be introduced.

- Chemical balance is solved with versatile chemical networks.

- Thermal balance is solved by considering the different heating and cooling processes. The photoelectric effect is calculated by including the extinction properties of the grains 
(silicates and carbon particles) and the actual computed value of the radiation field. The size distribution of dust particles is assumed to follow the Mathis, Rumpl \& Nordsieck (MRN) law (Mathis, Rumpl \& Nordsieck 1977). The cooling processes are determined via the emission of submillimeter and millimeter radiation of the main coolants following collisional excitation by $\mathrm{H}, \mathrm{He}, \mathrm{H}_{2} \ldots$

- The main shortcoming of such a model is the stationary approximation. However, the (photo)chemical time scales associated with diffuse regions are short, and such an assumption is reasonable.

The major input parameters and subsequent results are:

\begin{tabular}{ll}
\hline Parameters & Results \\
\hline $\begin{array}{l}\text { Equation of state: density, pressure, } \\
\text { temperature profile }\end{array}$ & $\begin{array}{l}\text { Abundance of the various species at } \\
\text { each point in } \mathrm{cm}^{-3}\end{array}$ \\
$\begin{array}{l}\text { Incident radiation field to the left and } \\
\text { right sides }\end{array}$ & $\begin{array}{l}\text { Excitation state of } \mathrm{C}^{+}, \mathrm{C}, \mathrm{O}, \ldots, \mathrm{H}_{2}, \\
\mathrm{HD}, \mathrm{CO}, \mathrm{CS}, \mathrm{HCO}^{+} \ldots\end{array}$ \\
Elemental abundances in the gas phase & $\begin{array}{l}\text { Individual heating and cooling } \\
\text { efficiencies in erg } \mathrm{cm}^{-3} \mathrm{~s}^{-1}\end{array}$ \\
Extinction curve, size distribution of grains & $\begin{array}{l}\text { Temperature profile of gas and grains } \\
\text { (per size) }\end{array}$ \\
$\begin{array}{l}\text { Cosmic ray ionisation rate, turbulent } \\
\text { velocity ... }\end{array}$ & $\begin{array}{l}\text { Integrated quantities (column densities, } \\
\text { emitting intensities of the "cloud") }\end{array}$ \\
$\begin{array}{l}\text { Atomic and molecular parameters } \\
\text { (Inelastic collision and chemical reaction }\end{array}$ & \\
rate coefficients ...) & \\
\hline
\end{tabular}

We define a standard cloud model with a density, $n_{\mathrm{H}}=100 \mathrm{~cm}^{-3}$, and a cosmic ionisation rate, $\zeta=5 \times 10^{-17} \mathrm{~s}^{-1}$, which is irradiated by the interstellar standard radiation field (ISRF) expressed in Draine's units $\dagger$ (the scaling factor $\chi=1$ ). The size of the cloud is defined by the total visual extinction value $A_{V}^{\text {tot }}=1$ corresponding to a colour excess $E_{B-V}$ of about 0.3 , representative of diffuse cloud conditions. The chemical network contains 120 species linked by 1900 chemical reactions, which are mainly taken from the most recent Ohio State University chemistry (cf. http://www. physics.ohiostate.edu/ eric/research.html).

\section{Discussion}

In the following, we discuss some common approximations performed in diffuse cloud calculations and test the role of different parameters.

\subsection{1-Sided versus 2-Sided Models}

For computing time reasons and numerical convenience, diffuse clouds are often represented as semi infinite slabs illuminated on one side. Then, column densities are obtained by integrating abundances up to $A_{\mathrm{V}}^{\text {tot }} / 2$ and multiplying the results by two. This assumption is justified when photochemical effects are negligible at this visual extinction. We test this hypothesis and compare in Table 1 the results obtained for three different representations of an interstellar cloud of total visual magnitude of 1 , immersed in the standard ultraviolet radiation field.

We have checked that the ionization fraction and the temperature profile are very similar in the range of visual magnitude between 0 and 0.5 . The derived column densities

$\dagger$ Cf. Draine 1978 
Table 1. Column densities $\left(\mathrm{cm}^{-2}\right)$ obtained for three different models.

\begin{tabular}{lccc}
\hline Molecule & $\mathbf{A}$ & $\mathbf{B}$ & $\mathbf{C}$ \\
\hline $\mathrm{H}$ & $2.2(20)$ & $2.3(20)$ & $1.4(20)$ \\
$\mathrm{H}_{2}$ & $8.2(20)$ & $8.1(20)$ & $8.6(20)$ \\
$\mathrm{C}$ & $1.3(15)$ & $1.9(15)$ & $8 .(15)$ \\
$\mathrm{CO}$ & $4.4(13)$ & $5.3(13)$ & $1.3(14)$ \\
$\mathrm{CH}$ & $4.1(13)$ & $5.2(13)$ & $7.5(13)$ \\
$\mathrm{C}_{2}$ & $7.8(12)$ & $1.7(13)$ & $6.1(13)$ \\
$\mathrm{C}_{3}$ & $9.4(11)$ & $3.2(12)$ & $1.6(13)$ \\
$\mathrm{OH}$ & $1.7(13)$ & $1.7(13)$ & $1.0(13)$ \\
\hline
\end{tabular}

Notes: Model A: the effect of the radiation field on the two sides of the cloud $\left(A_{\mathrm{V}}^{\text {tot }}=1\right)$ is computed. Model B: the radiation field is assumed to come from one side; the integration is performed until $A_{V}=0.5$ and the column densities are computed by multiplying the results by 2. Model C: the radiation field is assumed to come from one side; the integration is performed until $A_{V}=1$. Values in parenthesis refer to powers of ten.

of molecular hydrogen are marginally different in the 3 cases, since self-shielding effects are very efficient at low values of $A_{V}$. The results displayed above reflect the sensitivity of the various species to photoprocesses such as $\mathrm{CO}$, which is often used as a tracer of $\mathrm{H}_{2}$. The results also show, as known from previous studies, that $\mathrm{OH}$ is mainly formed in the external part of the cloud so that models A and B lead to similar values of the corresponding column density. Substantial differences are however obtained for carbon chain molecules such as $\mathrm{C}_{2}$ and $\mathrm{C}_{3}$ and it is therefore advisable to consider explicitly the two-sided illumination for these species.

\subsection{Role of the Shielding of $\mathrm{H}_{2}$}

Photodissociation of $\mathrm{H}_{2}$ occurs via discrete absorption in the Lyman and Werner band systems followed by emission in the continuum of the electronic ground state. This leads to very efficient self-shielding of $\mathrm{H}_{2}$, which may be evaluated by following the approximation of Federman, Glassgold \& Kwan (1979), known as the FGK approximation.

Table 2. Column densities $\left(\mathrm{cm}^{-2}\right)$ calculated with the FGK approximation and with the "exact" solution of the ultraviolet radiative transfer.

\begin{tabular}{ccc}
\hline Model & $\begin{array}{c}\text { FGK } \\
\text { self-shielding of } \mathrm{H}_{2}\end{array}$ & $\begin{array}{c}\text { "Exact" } \\
\text { full radiative transfer }\end{array}$ \\
\hline$f$ & 0.88 & 0.92 \\
\hline column density & & \\
\hline $\mathrm{C}$ & $1.3(15)$ & $2.1(15)$ \\
$\mathrm{CH}$ & $4.1(13)$ & $4.8(13)$ \\
$\mathrm{CN}$ & $2.0(11)$ & $2.2(11)$ \\
$\mathrm{CO}$ & $4.4(13)$ & $7.0(13)$ \\
$\mathrm{OH}$ & $1.7(13)$ & $1.4(13)$ \\
$\mathrm{CS}$ & $1.0(11)$ & $1.2(11)$ \\
$\mathrm{C}_{2}$ & $7.8(12)$ & $9.4(12)$ \\
$\mathrm{C}_{3}$ & $9.4(11)$ & $1.2(12)$ \\
\hline
\end{tabular}

Note: Values in parenthesis refer to powers of ten.

However, the numerous electronic bands of $\mathrm{H}_{2}$ may reduce the intensity of the radiation field considerably as they overlap between themselves and with other ultraviolet transitions such as those of $\mathrm{CO}, \mathrm{C}, \ldots$ This effect is seldom taken into account as it requires a 
full treatment of the radiative transfer. We test its relevance on the molecular fraction $f=\frac{2 N\left(\mathrm{H}_{2}\right)}{N(\mathrm{H})+2 N\left(\mathrm{H}_{2}\right)}$ and on the column densities of the various species displayed in Table 2 . The major effect is on CO (and C) for which we perform the explicit calculation of photodissociation probability. Other molecules whose photodissociation rates are computed from analytic expressions are indirectly affected. Figure 1 displays the total absorption of the radiation field in the $912-1100 \AA$ window computed in the two approaches.

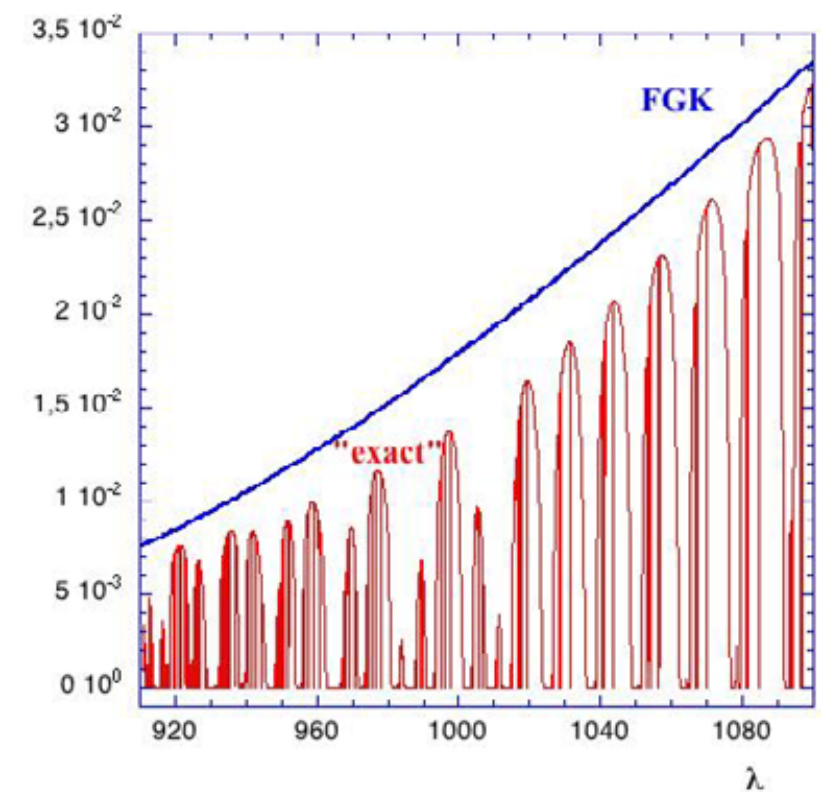

Figure 1. Total absorption factor of the radiation through the cloud

\subsection{Role of the Equation of State}

Constant density models are often considered, but isobaric conditions are more appropriate, as derived from the analysis of the populations of the atomic carbon fine structure levels (Jenkins 2002). The fractal structure of interstellar clouds is also a subject of discussion. Table 3 shows results for three models corresponding to different equations of states. The corresponding densities as a function of the visual extinction are displayed in Figure 2. Models B and C involve higher densities than model A. Then larger values of molecular column densities are obtained. Carbon chains are the most sensitive to density fluctuations.

\subsection{Chemical Issues}

\subsubsection{One Among Many Chemical Questions}

Atomic and molecular properties may also be considered as parameters. Not only are many reaction rate coefficients not available for interstellar temperature conditions, but radicals, reactive species found in the ISM are difficult to study in the laboratory. Then, a sensitivity analysis for the chemistry is an important issue ( $c f$. Roueff, Le Bourlot \& Pineau des Forêts 1996; Vasyunin et al. 2004). We address one single question amongst many others: this concerns the reaction between $\mathrm{H}$ and $\mathrm{CH}$. This reaction was supposed to have an activation energy of $\sim 2200 \mathrm{~K}$ (Aannestad 1973). However, recent theoretical 


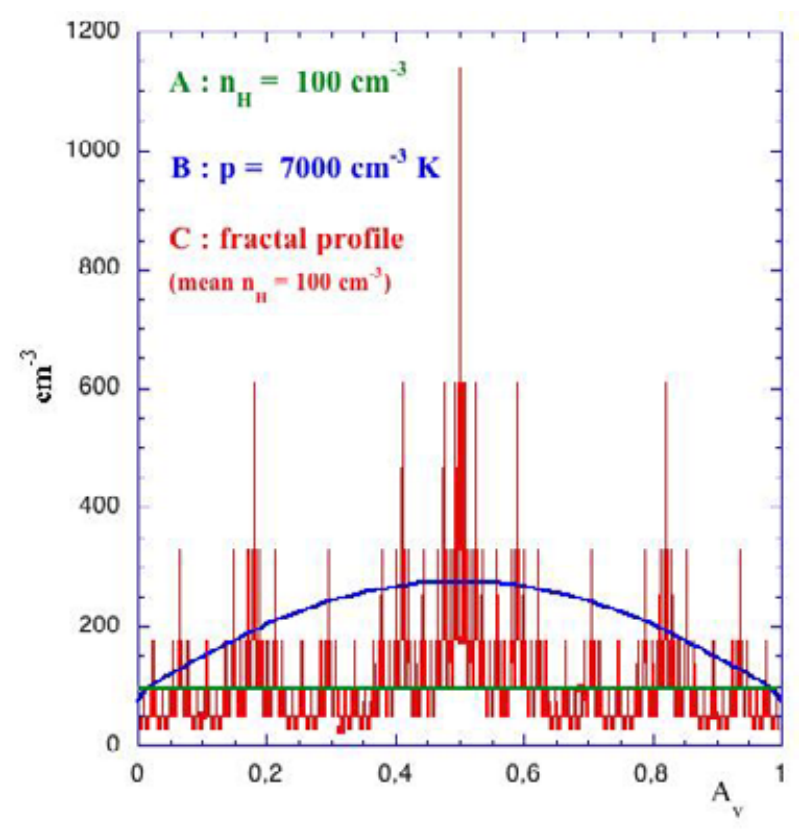

Figure 2. Density profiles in models A, B and C.

Table 3. Model A: $n_{\mathrm{H}}=100 \mathrm{~cm}^{-3}$. Model B: $p=7 \times 10^{3} \mathrm{~cm}^{-3} \mathrm{~K}$. Model C: fractal density profile with a mean value of $n_{\mathrm{H}}=100 \mathrm{~cm}^{-3}$.

\begin{tabular}{lccc}
\hline Models & A & B & C \\
\hline size $(\mathrm{pc})$ & 6.1 & 3.2 & 9.7 \\
mean value of $\mathrm{T}(\mathrm{K})$ & 80 & 57 & 81 \\
$f$ & 0.88 & 0.93 & 0.82 \\
\hline column density $\left(\mathrm{cm}^{-2}\right)$ & & & \\
$\mathrm{CO}$ & $4.4(13)$ & $6.3(13)$ & $3.6(13)$ \\
$\mathrm{CH}$ & $4.1(13)$ & $8.5(13)$ & $5.9(13)$ \\
$\mathrm{C}$ & $1.3(15)$ & $6.3(15)$ & $1.8(15)$ \\
$\mathrm{C}_{2}$ & $7.8(12)$ & $4.8(13)$ & $2.1(13)$ \\
$\mathrm{C}_{3}$ & $9.4(11)$ & $1.2(13)$ & $5.1(12)$ \\
$\mathrm{H}_{3}^{+}$ & $0.8(13)$ & $0.4(13)$ & $1.0(13)$ \\
\hline
\end{tabular}

Note: Values in parenthesis refer to powers of ten.

calculations by van Harrevelt, van Hemert \& Schatz (2002) find that this reaction should be rapid, and a discrepancy by a factor of 7 is found with the most recent experiment at $300 \mathrm{~K}$ (Becker et al. 1989). The question remains open. In diffuse cloud conditions, there is a competition between $\mathrm{CH}$ photodissociation, which is known accurately from ab-initio calculations by van Dishoeck (1987) and the reaction with $\mathrm{H}$, which may occur in the absence of any activation barrier.

\subsubsection{Empirical Introduction of Non-Thermal Effects}

The modelling of $\mathrm{CH}^{+}$has been a longstanding problem for diffuse cloud models since this ion is destroyed by both atomic and molecular hydrogen. In addition, the reaction of $\mathrm{C}^{+}$with $\mathrm{H}_{2}$ is endothermic by about $0.36 \mathrm{eV}$. Then, the computed abundance is smaller than the observations by more than 2 orders of magnitude in all quiescent interstellar 
Table 4. Effect on the "standard model" of the empirical introduction of non-thermal effects for ion-molecule reactions.

\begin{tabular}{lcccc}
\hline Models & standard & $\mathrm{v}_{i n}=2 \mathrm{~km} \mathrm{~s}^{-1}$ & $\mathrm{v}_{i n}=3 \mathrm{~km} \mathrm{~s}^{-1}$ & $\mathrm{v}_{i n}=4 \mathrm{~km} \mathrm{~s}^{-1}$ \\
\hline column density $\left(\mathrm{cm}^{-2}\right)$ & & & & \\
$\mathrm{CH}$ & $4.1(13)$ & $5.2(13)$ & $9.6(13)$ & $1.3(15)$ \\
$\mathrm{CN}$ & $2.0(11)$ & $1.9(11)$ & $5.9(12)$ & $2.1(13)$ \\
$\mathrm{CO}$ & $4.4(13)$ & $4.6(13)$ & $2.5(14)$ & $7.5(14)$ \\
$\mathrm{OH}$ & $1.7(13)$ & $3.6(13)$ & $8.0(13)$ & $1.5(14)$ \\
$\mathrm{C}_{2}$ & $7.8(12)$ & $2.9(12)$ & $3.8(13)$ & $3.6(13)$ \\
$\mathrm{H}_{3}^{+}$ & $8.3(12)$ & $8.5(12)$ & $5.4(12)$ & $1.4(12)$ \\
$\mathrm{CH}^{+}$ & $1.6(11)$ & $1.9(11)$ & $3.2(13)$ & $2.4(14)$ \\
$\mathrm{HCO}^{+}$ & $7.0(10)$ & $6.0(10)$ & $2.8(11)$ & $1.1(12)$ \\
\hline
\end{tabular}

Note: Values in parenthesis refer to powers of ten.

models. To allow this reaction to proceed, various hypothesis on energy inputs have been proposed, among them: reaction with vibrationally excited $\mathrm{H}_{2}$ (Garrod et al. 2003), MHD shocks (Pineau des Forêts et al. 1986), intermittent turbulent energy (Falgarone, Pineau des Forêts \& Roueff 1995; Joulain et al. 1998; Falgarone et al., this conference). A simple formulation to include non thermal effects was given by Federman et al. (1996), where an effective temperature, defined for each ion-neutral reaction, as $\frac{3}{2} \mathrm{k} T_{\text {eff }}=\frac{3}{2} \mathrm{k} T_{k i n}+$ $\frac{1}{2} \mu v_{i n}^{2}$, is used in chemical reactions rates. In this expression, $\mathrm{v}_{i n}$ is the relative ion-neutral drift speed and $\mu$ the reduced mass of the system. In Table 4 we compare models for different values of the ion-neutral drift velocity.

We note that this modus operandi allows us indeed to increase significantly the amount of predicted $\mathrm{CH}^{+}$and to a lesser extent $\mathrm{CO}, \mathrm{OH}$ and $\mathrm{HCO}^{+}$. The values obtained for an ion-neutral drift velocity of $3 \mathrm{~km} \mathrm{~s}^{-1}$ are close to the observed order of magnitudes of $\mathrm{N}\left(\mathrm{CH}^{+}\right)$. However, this procedure is empirical and does not account for the actual physical processes at work, contrary to what has been done by Joulain et al. (1998).

\subsection{Role of the Cosmic Ionization Rate}

The recent detection of $\mathrm{H}_{3}^{+}$towards the diffuse $\zeta$ Per line of sight by McCall et al. (2003) has been explained by an enhancement of the cosmic ionization rate, $\zeta$, by 2 orders

Table 5. Role of the cosmic ionization rate $\zeta$ on the "standard model" conditions compared to the observed values towards $\zeta$ Per.

\begin{tabular}{lcccccc}
\hline$\zeta\left(\mathrm{s}^{-1}\right)$ & $\begin{array}{c}5 \times 10^{-17} \\
(\text { standard })\end{array}$ & $1 \times 10^{-16}$ & $5 \times 10^{-16}$ & $1 \times 10^{-15}$ & $5 \times 10^{-15}$ & $\zeta$ Per \\
\hline $\mathrm{f}$ & 0.88 & 0.87 & 0.76 & 0.66 & 0.36 & $0.53-0.66$ \\
$\mathrm{~T}(\mathrm{~K})$ & 80 & 71 & 69 & 67 & 68 & $45-75$ \\
\hline column density & $\left(\mathrm{cm}^{-2}\right)$ & & & & & \\
\hline $\mathrm{CH}$ & $4.1(13)$ & $3.4(13)$ & $1.2(13)$ & $5.8(12)$ & $9.0(11)$ & $1.9(13)-2(13)$ \\
$\mathrm{CN}$ & $2.0(11)$ & $2.2(11)$ & $2.4(11)$ & $2.2(11)$ & $4.4(10)$ & $2.7(12)-3.3(12)$ \\
$\mathrm{CO}$ & $4.4(13)$ & $6.4(13)$ & $1.8(14)$ & $1.4(14)$ & $2.8(13)$ & $5.4(14)$ \\
$\mathrm{OH}$ & $1.7(13)$ & $2.5(13)$ & $6.0(13)$ & $4.8(13)$ & $1.6(13)$ & $4.0(13)$ \\
$\mathrm{C}_{2}$ & $7.8(12)$ & $6.3(12)$ & $1.3(12)$ & $4.0(11)$ & $1.5(10)$ & $1.6(13)$ \\
$\mathrm{C}_{3}$ & $0.9(12)$ & $0.7(12)$ & $0.6(12)$ & $7.0(9)$ & $5.3(6)$ & $1.0(12)$ \\
$\mathrm{H}_{3}^{+}$ & $8.3(12)$ & $1.4(13)$ & $3.0(13)$ & $3.0(13)$ & $1.8(13)$ & $8 .(13)$ \\
$\mathrm{CH}^{+}$ & $1.6(11)$ & $1.7(11)$ & $1.6(11)$ & $1.4(11)$ & $6.7(10)$ & $3.5(12)$ \\
$\mathrm{HCO}^{+}$ & $7.0(10)$ & $8.610)$ & $1.1(11)$ & $5.2(10)$ & $3.2(9)$ & \\
& & & & & &
\end{tabular}

Note: Values in parenthesis refer to powers of ten. 
of magnitude on the basis of its formation and destruction processes. We have tested this assumption with our chemical network and display in Table 5 the column densities for different values of $\zeta$. We see that the $\mathrm{H}_{3}^{+}$column density increases with the cosmic ionization rate until the latter reaches a value of $5 \times 10^{-16} \mathrm{~s}^{-1}$. We find however that for higher values of the cosmic ionization rate, the molecular fraction becomes too low to account for the observed $\mathrm{H}_{3}^{+}$and that the column density of all molecular species decrease dramatically, in contradiction to the finding of McCall et al. (2003).

\section{Comparison with Observations}

\subsection{Excitation of $\mathrm{H}_{2}$}

We discuss now the problem of the rotational excitation of $\mathrm{H}_{2}$. Figure 3 displays the $\mathrm{H}_{2}$ excitation diagram for different lines of sight, as well as for models with different values of the scaling factor, $\chi_{l e f t}$, of the radiation field impinging the left side of the "cloud". The lines of sight include Copernicus data ( $\zeta$ Oph and $\zeta$ Per), high galactic clouds in the Chameleon (HD 96675, HD 102065 and HD 108927), which have been studied with FUSE (Gry et al. 2002), HD 37903 which corresponds to a cloud illuminated by the bright $\theta$ Ori star (Meyer et al. 2001) and HD 34078 (Boissé et al. 2005). We note that the highest rotational levels are not well reproduced by any homogeneous model, even with radiation fields 10 times the standard values. Moreover, a high value of $\chi$ leads to a substantial increase of the temperature and the ratio between $J=0$ and 1 rotational levels is no more satisfactory. Other processes involving external input of energy can be at work and lead to excitation of molecular hydrogen as well as formation of $\mathrm{CH}^{+}$. MHD shock models ( $c f$. Heck et al. 1993) and turbulent chemical models of dissipative diffuse structures (Falgarone et al. 2005) are promising in this respect. However, the coupling of these macroscopic mechanisms with the detailed UV radiative transfer driving the atomic to molecular transition has not yet been fully introduced.

\subsection{The $\zeta$ Per Line of Sight}

The discovery of $\mathrm{H}_{3}^{+}$by McCall et al. (2003) towards $\zeta$ Per has led Le Petit, Roueff \& Herbst (2004) to consider all observational constraints available in a chemical model, using the Meudon PDR code. It is clear from this study that a single density component is not able to reproduce the observations. The combination of a diffuse $\left(n_{\mathrm{H}}=100 \mathrm{~cm}^{-3}\right.$ with $A_{V}=0.69$ corresponding to $\left.4.2 \mathrm{pc}\right)$ and dense slab $\left(n_{\mathrm{H}}=2 \times 10^{4} \mathrm{~cm}^{-3}\right.$ with $A_{V}$ $=0.013$ corresponding to $76 \mathrm{AU}$ ) model is required to fit both atomic and molecular column densities in a reasonable way. A cosmic ray ionization rate of $2.5 \times 10^{-16} \mathrm{~s}^{-1}$ is derived. The column density of $\mathrm{H}_{3}^{+}$is reproduced within a factor of 3 . Similar conclusions had been formerly obtained by Cecchi-Pestellini \& Dalgarno (2002) in the analysis of $\mathrm{C}_{2}$ towards diffuse interstellar clouds. Introducing density fluctuations, length-scales and filling factors could be important for the interpretation of diffuse cloud chemistry. It should be noted that the excitation of rotational levels of $\mathrm{H}_{2}$ above $J=2$ and the observed column density of $\mathrm{CH}^{+}$are not reproduced in such a 2-phase model. An additional energetic phase is required for these additional observational constraints.

\section{Conclusions}

We have studied the dependence of a steady-state diffuse cloud model on various physical and astrophysical parameters. Much care has been paid to detailed microphysical processes. Carbon chains appear to be very sensitive to various parameters such as density profiles and geometry (one-sided or two-sided irradiation). The influence of cosmic ray 


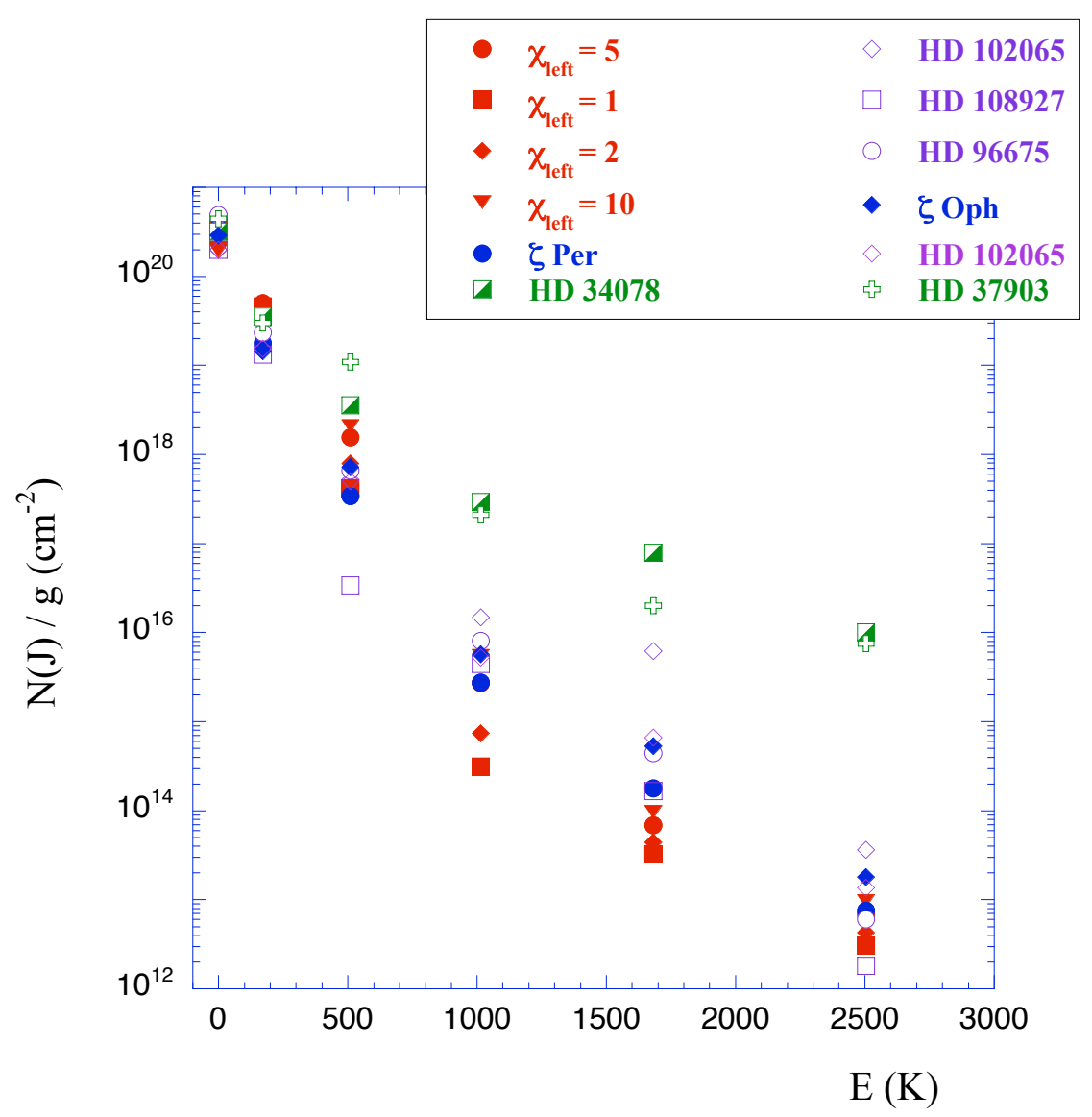

Figure 3. Rotational diagram of $\mathrm{H}_{2}$.

ionization is decisive on the chemistry of $\mathrm{H}_{3}^{+}$. However, too large a value for the cosmic ionization rate has a destructive impact on the molecular composition (via the secondary UV photons in particular) and the modelled column density of this molecular ion remains a factor 2 to 3 below the observed value.

Present steady-state models appear unsatisfactory to explain the large variety of observations in diffuse environments. However, such preliminary detailed studies are mandatory before introducing specific processes dealing with the astrophysical environment (time dependence, density fluctuations, MHD shocks, turbulence, ...). In spite of many observations and models, the chemistry of diffuse regions remains elusive.

\section{Acknowledgements}

We would like to acknowledge the many discussions we had with J. Le Bourlot and the support from the French Program "Physico-Chimie du Milieu Interstellaire" (PCMI).

\section{References}

Aannestad, P. 1973, Ap. J. Suppl. 25, 223

Abgrall, H., Le Bourlot, J., Pineau des Forêts G., Roueff, E., Flower, D.R., \& Heck, L. 1992, $A \mathscr{E} A 253,525$ 
Becker, K.H., Engelhardt, B., Wiesen, P., Bayes, K.D. 1989, CPL 154, 342

Boissé, P., Le Petit, F., Rollinde, E., Roueff, E., Pineau des Forêts, G., Andersson, B.G., Gry, C., \& Felenbok, P. 2005, A\&A 429, 509

Cecchi-Pestillini, C., \& Dalgarno, A. 2002 MNRAS 334, L31

Draine, B.T. 1978, Ap. J. Suppl. 36, 595

Falgarone, E., Pineau des Forêts, G., \& Roueff, E. 1995, A\&̇A 300, 870

Falgarone, E., Verstraete, L., Pineau des Forêts, G., \& Hily-Blant, P. 2005, A $\& A$ 433, 997

Falgarone, E., Hily-Blant, P., Pineau des Forêts, G., \& Schilke, P. 2005, IAUS231, poster I.21

Federman, S.R., Glassgold, A.E., \& Kwan J. 1979, Ap. J. 227, 466

Federman, S.R., Rawlings, J.M.C., Taylor, S.D., \& Williams D.A. 1996, MNRAS 279, L41

Garrod, R.T., Rawlings, J.M.C., Williams, D.A. 2003, ApESSS 286, 487

Gry, C., Boulanger, F., Nehmé, C., Pineau des Forêts, G., Habart, E., \& Falgarone, E. 2002, A\&A 391,675

Heck, E.L., Flower, D.R., Le Bourlot, J., Pineau des Forêts, G., \& Roueff, E. 1993, MNRAS 258,233

Jenkins, E.B. 2002, Ap. J. 580, 938

Joulain, K., Falgarone, E., Pineau des Forêts, \& Flower, D.R. 1998, A\&A 340, 241

Knauth, D.C., Andersson, B.-G., McCandiss S.R., \& Warren Moos, H. 2004, Nature 429, 636

Le Bourlot, J., Pineau des Forêts G., Roueff, E., \& Flower, D.R. 1993, A\&̈A 267, 233

Le Petit, F., Roueff, E., \& Herbst, E. 2004, A\&A 417, 993

Le Petit, F., Nehmé, C., Hily-Blant P., Le Bourlot, J., Roueff, E., \& Le Bourlot, E. 2005, A\&A, to be submitted

Liszt, H., Lucas, R., \& Black, J.H. 2004, A\&A 428, 117

Mathis, J. S., Rumpl, W., \& Nordsieck, K.H. 1977, Ap. J., 217, 425

McCall, B.J. et al. 2003, Nature 422, 500

Meyer, D.M., Lauroesch, J.T., Sofia, U.J., Draine, B.T. \& Bertoldi, F. 2001, Ap. J. 553, L59

Pineau des Forêts, G., Flower, D.R., Hartquist, T.W. , \& Dalgarno, A. 1986, MNRAS 230, 801

Roueff, E., Le Bourlot, J., \& Pineau des Forêts G. 1996, in Dissociative Recombination: Theory, Experiment and Applications, rds. D. Zajfman, J.B.A. Mitchell, D. Schwalm, \& B.R. Rowe (World Scientific, Singapore), 11

van Harrevelt, R., van Hemert, M.C., \& Schatz, G.C. 2002 , JCP 116, 6002

van Dishoeck, E. F. 1987, JCP, 86, 196

Vasyunin, A.I., Sobolev, A.M., Wiebe, D.S. and Semenov D.A. 2004, Astr. Letters, 30, 566

\section{Discussion}

VAN Dishoeck: You have not said much about HD, whose abundance is also sensitive to the cosmic ray ionization rate. Can you comment?

RouefF: A promising way to determine HD column densities was indeed via observations in the far UV in absorption (Copernicus, FUSE). However, the accurate determination of HD column density is hampered by saturation effects because the corresponding transitions lie in the flat portion of the equivalent width. The column density of HD is then determined to within a factor 5-10 only and does not constrain model results.

Bensch: The 2-phase model for $\zeta$ Per has a much higher CO column density in the diffuse component than in the dense component, and the diffuse models shown before for $A_{V}=1$. Why is the CO column density here (for $X=2, A_{V}<1$ ) much higher than shown before for $X=1, A_{V}=1$ ?

RouefF: The CO is indeed produced in the diffuse component: the higher cosmic ionization rate aids the formation of $\mathrm{CO}$ as $\mathrm{OH}$ is substantially increased and the $\mathrm{C}^{+}+\mathrm{OH}$ reaction is the main channel to $\mathrm{CO}$ production. 
NEUfELD: What happens if you throw up your hands, say that there is something we simply don't understand about $\mathrm{H}_{3}^{+}$, and put in the observed abundance into the models by hand (i.e. treat the $\mathrm{H}_{3}^{+}$abundance as a fixed parameter rather than a quantity obtained by solution of the rate equations)? What problems would this solve in diffuse cloud chemistry (and what problems might it create)?

RouEFF: This is an interesting suggestion and would probably help to build polyatomic species - more detailed consequences deserve further study.

MCCALL: I have two concerns with the dense component included in your model of zeta Persei. First, the observed rotational distribution of $\mathrm{C}_{2}$ and $\mathrm{C}_{3}$ seems to be inconsistent with such a high density as $20,000 \mathrm{~cm}^{-3}$. Second, if the size of the densest cloudlet on the sky were comparable to its supposed length (76 AU), it seems quite lucky that we happen to be looking through it. If we wait for a couple of years, it will likely no longer be along the line of sight, due to proper motion. Do you have any comment on these concerns?

RouefF: Your question refers to the results reported in the paper by Le Petit, Roueff $\&$ Herbst (2004). Concerning your first concern, we have run your code available at the URL http://dib.uiuc.edu/c2/ computing the excitation of $\mathrm{C}_{2}$ to check the relevance of a dense component, and we found that such a solution was indeed compatible with the observations except for $\mathrm{J}=6$. However, a similar problem arises for a lower density component such as one with density $100 \mathrm{~cm}^{-3}$. The observed $J=6$ column density may be over-estimated. Indeed, only one transition, $\mathrm{R}(6)$, has been found whereas the two others, $\mathrm{P}(6)$ and $\mathrm{Q}(6)$, have not been detected.

Your argument about possible small scale structure is perfectly reasonable. However, there is mounting observational evidence that interstellar matter can have a clumpy or filamentary structure on the scale of 10s to a few 1000s of AU and Cordiner, Fossey and Sarre (this conference) report such occurrences from diffuse band strength studies. Such an hypothesis is thus in accordance with present observational knowledge. 